\title{
Relaxed State and Fault Estimation for Vehicle Lateral Dynamics Represented by T-S Fuzzy Systems
}

\author{
N. El Youssfi (iD, R. El Bachtiri, T. Zoulagh, and H. El Aiss \\ Industrial Technologies and Services Laboratory (Lab-TSI), Higher School of Technology, STI-FST Doctoral Study Centre, \\ Sidi Mohamed Ben Abdellah University, Fez, Morocco
}

Correspondence should be addressed to N. El Youssfi; naoufal.elyoussfi@usmba.ac.ma

Received 13 January 2020; Accepted 24 August 2020; Published 12 September 2020

Academic Editor: Radek Matušů

Copyright (C) 2020 N. El Youssfi et al. This is an open access article distributed under the Creative Commons Attribution License, which permits unrestricted use, distribution, and reproduction in any medium, provided the original work is properly cited.

This paper deals with the problem of observer design of the vehicle model which is represented by Takagi-Sugeno (T-S) fuzzy systems with the presence of uncertainties. A relaxed observer design is presented to estimate the unmeasurable states and the faults of the vehicle lateral dynamics model, simultaneously. The vehicle model is transformed into a system with unknown inputs. Then, it is rebuilt by adding the default to the system state equation. Based on the Lyapunov function approach and the introduction of some slack variables, sufficient conditions of unknown input observer design are formulated as Linear Matrix Inequalities (LMIs). Finally, the simulation section clearly shows the importance and effectiveness of the proposed strategy.

\section{Introduction}

A large part of the scientific research programs in the transport field is focused on road safety, to respond to the problems related to the evolution of the transport means. Today's vehicles are becoming more intelligent, reliable, relaxed, and safe because they are equipped with various security systems, either passive as Bumper, whose classic function is to protect the bodywork. But, bumpers today play a bigger role. They have become very safe and aim, above all, to protect pedestrians and cyclists in the event of an accident $[1,2]$. Seat belts are among the indispensable passive safety devices. They protect vehicle occupants in a potentially dangerous collision. They were proposed by the American car manufacturer Nash in 1949 as a new option and were developed in their modern form by the Swedish inventor Nils Bohlin for Volvo [3, 4]. We also mention airbags; they are considered essential equipment and have proven several times that they can save lives. The first ones were tested by General Motors in 1973 on the Chevrolet model $[5,6]$ or active as the Antilock Braking System (ABS), which prevents the wheels from blocking and, thus, the vehicle skidding during braking; it was first marketed by Bosch in the 1970s $[7,8]$. The Electronic Stability Program (ESP) was developed in the 1990s by Bosch exclusively for the Mercedes S-Class $[9,10]$, and this technology makes it possible to avoid a road trip in extreme situations via selective and individual braking of the wheels $[11,12]$. These systems are generally controlled by both known and unknown inputs. Their functioning and efficiency require precise knowledge of the dynamic parameters of the vehicle. Sometimes, the measurements of these parameters do not give complete information about the system because some states are not directly measurable. In addition, for cost reasons, the number of sensors is limited and is sometimes unavailable. The idea is to use a software sensor or an observer capable of reconstructing state information, unmeasurable parameters, and even unknown inputs of the system from the system model and measured parameters.

In this context, different estimation techniques have been applied to resolve observer design problems. Among these techniques, we can find Luenberger's classical observer $[13,14]$, which is based on the synthesis of a static gain, to ensure the convergence of the observer's states towards the real states of the system and to stabilize the estimation error. However, the presence of perturbations on the system leads to a bad reconstruction. Also, the Kalman filter (KF) $[15,16]$ is robust against measurement noise. Another example is the 
Sliding Mode Observer (SMO) $[17,18]$, which is characterized by asymptotic decay of the state estimation error even in the presence of uncertainties in the system input and nonlinearities. The quoted observers can no longer be applied when the dynamics of the system are subject to the influence of unknown inputs. Generally, these unknown inputs come from sensor faults, modeling errors, disturbances, or noise [19]. To overcome these limits, the Unknown Input Observer (UIO) is widely studied in the literature [20-22], and it makes it possible to reconstruct system states even in the presence of unknown inputs and often intervenes in diagnosis, for fault detection, sensor monitoring, and estimation of disturbances affecting the system.

For this purpose, our main objective in this manuscript is to accurately estimate the lateral dynamics of the vehicle with the presence of uncertainties and sensor/actuator faults by a relaxed unknown inputs observer. The best-known model used to describe the lateral movements of the vehicle is the bicycle model, developed in [23], which will be represented by the Takagi-Sugeno (T-S) multiple-model approach, widely used in the literature to solve nonlinearity problems in nonlinear systems [24-26], which consists in developing the global model by interpolation of local linear models. This technique accurately describes the behavior of nonlinear systems, including the vehicle's lateral dynamics system $[27,28]$. The stability of the estimation error toward zero is mainly studied by using the Lyapunov quadratic function, and sufficient asymptotic stability conditions are given in the form of linear matrix inequalities (LMIs), which can be solved very effectively using optimization techniques of LMI [29].

The structure of the document is organized as follows: Section 2 deals with the model of vehicle lateral dynamics, as well as its T-S fuzzy representation. Section 3 describes the problem. Section 4 presents the estimation error stability analysis and the design of a relaxed unknown inputs observer. Section 5 is devoted to simulations and results analysis. Section 6 is devoted to discussion. Finally, the conclusion is given in the Section 7 .

Notations. The following notations are considered: $\mathbb{R}^{n}$ means the $n$-dimensional Euclidean space, and $I$ is the identity matrix with appropriate dimension. The notations $A<0$ and $A \leq 0$ indicate that the matrix $A$ is negatively defined and negatively semidefined, respectively. The inverse of matrix $A$ is expressed by $A^{-1}$, and its transpose is indicated by $A^{T} . *$ means symmetrical terms in a symmetrical matrix. $A^{\dagger}$ denotes a generalized inverse of $A$.

\section{Vehicle Modelling}

2.1. Nonlinear Vehicle Model. The complete vehicle dynamics model is studied in [30], which is very difficult to use in control and monitoring applications because it is a very complex system and has many freedom degrees. For this reason, a simplified model that is easy to use to the synthesis of observers and controls is indispensable. The vehicle motions are defined by a set of translations and rotational movements illustrated to the top left of Figure 1 [28]. The model used in this paper describes the vehicle lateral dynamics (see Figure 1), which is obtained by considering the bicycle model; the lateral velocity $v_{y}$ and the yaw rate $\dot{\psi}$ of the vehicle are taken to be differential variables.

The lateral dynamics of the vehicle is presented as in [23] by the following differential equations:

$$
\left\{\begin{array}{l}
\dot{v}_{y}=\frac{1}{m_{v}}\left(2 F_{y f}+2 F_{y r}\right)-v_{x} \dot{\psi}, \\
\ddot{\psi}=\frac{1}{I_{z}}\left(2 a_{f} F_{y f}+2 a_{r} F_{y r}+M_{z}\right),
\end{array}\right.
$$

where $v_{x}$ and $v_{y}$ are longitudinal and lateral velocities, respectively. $\dot{\psi}$ is the yaw rate, $m_{v}$ is the vehicle mass, $M_{z}$ is the external yaw moment, and $I_{z}$ is inertia moments around vertical axis. $F_{y f}$ and $F_{y r}$ are lateral tire forces at the front and the rear wheels, respectively. For further explanation of the variables appearing in the vehicle lateral dynamics model, refer to Table 1 and Figure 1.

\subsection{T-S Fuzzy Representation for Vehicle Lateral Dynamics.} A T-S fuzzy model is a set of linear time-invariant (LTI) systems, blended with nonlinear membership functions. Different ways to perform a T-S model from nonlinear models exist. An interesting approach is the well-known nonlinear sector transformation [31]. In fact, this technique allows obtaining an exact $\mathrm{T}-\mathrm{S}$ representation without information loss on a compact set of the state space. By using identification and linearization of the cornering forces on the vehicle which can be approximated as in $[20,27]$, they are given by the following expressions:

$$
\left\{\begin{array}{l}
F_{y f}(t)=\sum_{i=1}^{2} \rho_{i}(\xi(t)) C_{f i} \alpha_{f}(t), \\
F_{y r}(t)=\sum_{i=1}^{2} \rho_{i}(\xi(t)) C_{r i} \alpha_{r}(t),
\end{array}\right.
$$

where $C_{f i}$ and $C_{r i}$ are the front and rear tire cornering stiffness which depend of road adhesion and vehicle mass $m_{v}$ and $\alpha_{f}$ and $\alpha_{r}$ are slip angles of front and rear tires, respectively. They are given in [32] as follows:

$$
\left\{\begin{array}{l}
\alpha_{f}(t)=\delta_{f}(t)-\beta(t)-\frac{a_{f} \dot{\psi}(t)}{v_{x}}, \\
\alpha_{r}(t)=-\beta(t)-\frac{a_{r} \dot{\psi}(t)}{v_{x}}
\end{array}\right.
$$

where $\beta(t)=\left(v_{y}(t) / v_{x}(t)\right)$ is the sideslip angle. Membership functions $\rho_{i}(\xi(t))$ are given as follows:

$$
\rho_{i}(\xi(t))=\frac{\omega_{i}(\xi(t))}{\sum_{i=1}^{2} \omega_{i}(\xi(t))}, \quad i=1,2,
$$

with 


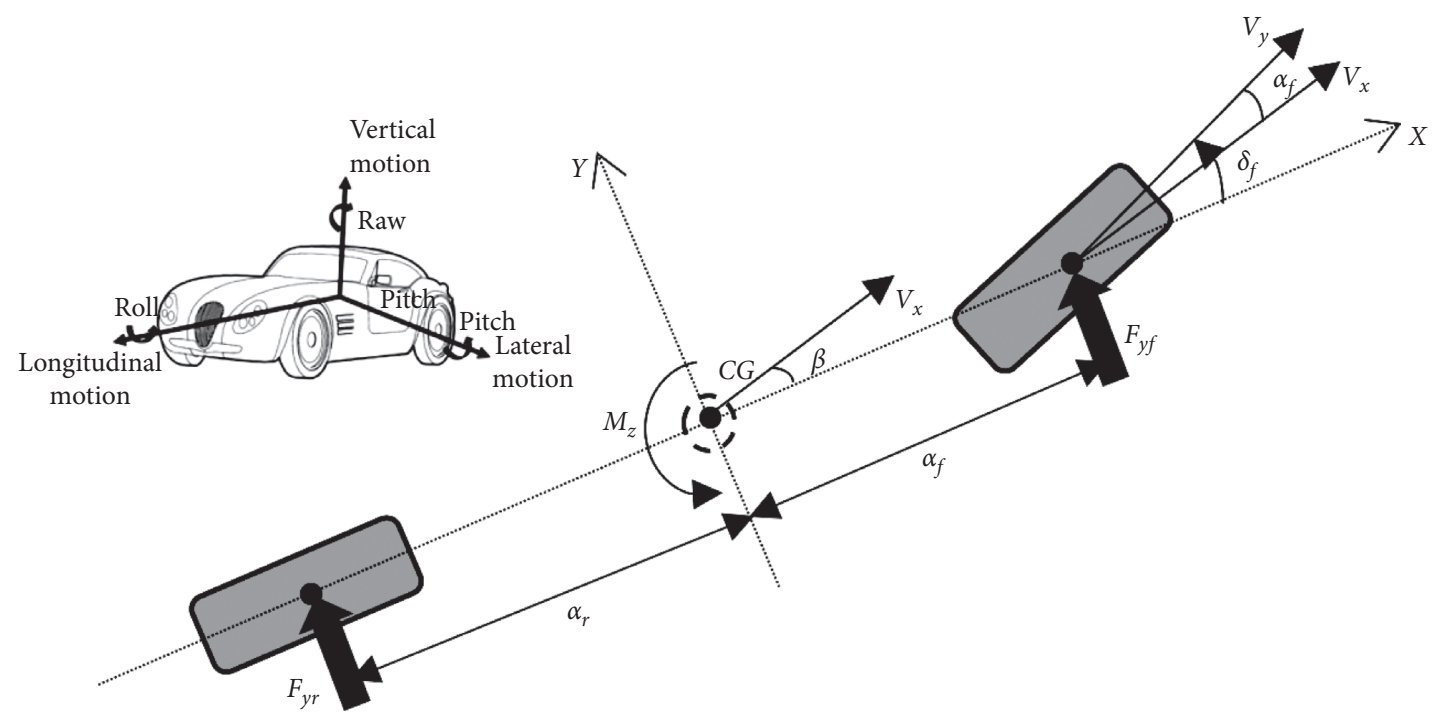

FIgURE 1: Vehicle lateral dynamics model.

TABLE 1: Simulation vehicle parameters.

\begin{tabular}{|c|c|c|c|}
\hline Constant & Explanation & Value & Unit \\
\hline$m$ & Vehicle mass & $1653+2 \times 90$ & $\mathrm{Kg}$ \\
\hline$I_{z}$ & Yaw inertia moment at the center of gravity & 2765 & $\mathrm{Kg} \cdot \mathrm{m}^{2}$ \\
\hline$a_{f}$ & Distance from the center of gravity to the front axle & 1.402 & $\mathrm{~m}$ \\
\hline$a_{r}$ & Distance from the center of gravity to the rear axle & 1.646 & $\mathrm{~m}$ \\
\hline
\end{tabular}

TABLE 2: Nominal stiffness and membership function coefficients.

\begin{tabular}{lccccccc}
\hline Nominal stiffness coefficients & $C_{f 1}$ & $C_{f 2}$ & $C_{r 1}$ & $C_{r 2}$ & $a_{1}=3.1893$ & $b_{1}=0.5077$ & $c_{1}=0.9496$ \\
Values & 60712 & 4812 & 60088 & 4555 & $a_{2}=0.5633$ & $b_{2}=5.3907$ & $c_{2}=0.8712$ \\
\hline
\end{tabular}

$$
\omega_{i}(\xi(t))=\frac{1}{\left(1+\left|\xi(t)-c_{i} / a_{i}\right|\right)^{2 b_{i}}}, \quad \xi(t)=\left|a_{f}(t)\right|
$$

and they satisfy the following properties:

$$
\left\{\begin{array}{l}
\sum_{i=1}^{2} \rho_{i}(\xi(t))=1 \\
0 \leq \rho_{i}(\xi(t)) \leq 1, \quad i=1,2
\end{array}\right.
$$

Membership function parameters $\left(a_{i}, b_{i}\right.$, and $\left.c_{i}\right)$ and stiffness coefficient values $\left(C_{f i}\right.$ and $\left.C_{r i}\right)$ are obtained using a Levenberg-Marquardt algorithm-based identification method combined with the least square method [33]. The T-S fuzzy model of vehicle lateral dynamics is obtained by replacing the lateral forces in the nonlinear model (1) by their fuzzy expressions (2). Then, lateral motion can be expressed by

$$
\left\{\begin{array}{l}
\dot{x}(t)=\sum_{i=1}^{2} \rho_{i}(\xi(t))\left\{A_{i} x(t)+B_{f i} \delta_{f}(t)+B M_{z}\right\}, \\
y(t)=C x(t)
\end{array}\right.
$$

where $x(t)$ is the system state vector, $y(t)$ is the system output vector, and $\delta_{f}(t)$ is the steering angle given by the driver. $A_{i}$, $B_{f i}, B$, and $C$ are constant matrices with compatible dimensions.

$$
\begin{aligned}
A_{i} & =\left[\begin{array}{cc}
-2 \frac{C_{f i}+C_{r i}}{m_{v} v_{x}} & -v_{x}-2 \frac{a_{f} C_{f i}-a_{r} C_{r i}}{m_{v} v_{x}} \\
-2 \frac{a_{f} C_{f i}-a_{r} C_{r i}}{I_{z} v_{x}} & -2 \frac{a_{f}^{2} C_{f i}+a_{r}^{2} C_{r i}}{I_{z} v_{x}}
\end{array}\right], \\
B_{f i} & =\left[\begin{array}{c}
2 \frac{C_{f i}}{m_{v} v_{x}} \\
2 \frac{a_{f} C_{f i}}{I_{z}}
\end{array}\right], \\
B & =\left[\begin{array}{l}
0 \\
1 \\
I_{z}
\end{array}\right], \\
C & =\left[\begin{array}{ll}
1 & 0 \\
0 & 1
\end{array}\right] .
\end{aligned}
$$

\section{Problem Description}

Vehicle lateral dynamics may show an unexpected dangerous behavior in the presence of unusual external conditions such as lateral wind force and the variation of the 
road adhesion coefficient. So, to deal with this problem, sensor/actuator faults and appropriate uncertainties must be introduced. For that, the uncertain T-S fuzzy system is considered as follows:

$$
\left\{\begin{array}{l}
\dot{x}(t)=\sum_{i=1}^{2} \rho_{i}(\xi(t))\left\{A_{i} x(t)+\Delta A_{i}(t) x(t)+B_{f i} \delta_{f}(t)+\Delta B_{f i}(t) \delta_{f}(t)+B M_{z}(t)+D_{i} f(t)\right\}, \\
y(t)=C x(t)+F f(t),
\end{array}\right.
$$

where $f(t)$ is faults affected to both the actuator and sensor. $D_{i}$ and $F$ are constant matrices with compatible dimensions. $\Delta A_{i}(t)$ and $\Delta B_{f i}(t)$ are matrices functions which represent time-varying parameter uncertainties affecting the state and the input, respectively, and are structured as follows:

$$
\begin{aligned}
\Delta A_{i}(t) & =M \Delta \widetilde{A}_{i}(t), \\
\Delta B_{f i}(t) & =M \Delta \widetilde{B}_{f i}(t),
\end{aligned}
$$

where $M$ is a full column rank matrix.

Due to uncertainties (10), the T-S model (9) can be rewritten as

$$
\left\{\begin{array}{l}
\dot{x}(t)=\sum_{i=1}^{2} \rho_{i}(\xi(t))\left\{A_{i} x(t)+B_{f i} \delta_{f}(t)+B M_{z}(t)+D_{i} f(t)+M\left(\Delta \widetilde{A}_{i}(t) x(t)+\Delta \widetilde{B}_{f i}(t) \delta_{f}(t)\right)\right\} \\
y(t)=C x(t)+F f(t) .
\end{array}\right.
$$

Let us put the following equalities:

$$
\left.\begin{array}{l}
h_{i}=\Delta \widetilde{A}_{i}(t) x(t) \\
k_{i}=\Delta \widetilde{B}_{f i}(t) \delta_{f}(t)
\end{array}\right\} \longrightarrow v_{i}(t)=k_{i}(t)+h_{i}(t),
$$

and then, by replacing $v_{i}(t)$ in (11), the system becomes as follows:

$$
\left\{\begin{array}{l}
\dot{x}(t)=\sum_{i=1}^{2} \rho_{i}(\xi(t))\left\{A_{i} x(t)+B_{f i} \delta_{f}(t)+B M_{z}(t)+M v_{i}(t)+D_{i} f(t)\right\} \\
y(t)=C x(t)+F f(t) .
\end{array}\right.
$$

The augmented system formed from system (13) and fault $f(t)$ can be expressed as

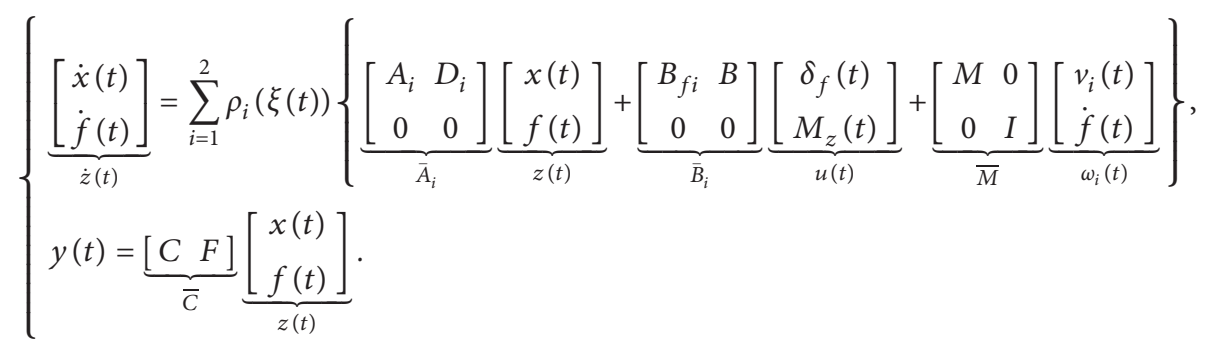

Then, we obtain

$$
\left\{\begin{array}{l}
\dot{z}(t)=\sum_{i=1}^{2} \rho_{i}(\xi(t))\left\{\bar{A}_{i} z(t)+\bar{B}_{i} u(t)+\bar{M} \omega_{i}(t)\right\} \\
y(t)=\bar{C} z(t)
\end{array}\right.
$$

Before moving on to designing the observer for the system (15), to estimate unmeasurable states and faults in the next section, the following assumptions and lemmas are needed.

Assumption 1 (see [22]). The matrices $\bar{C}$ and $\bar{M}$ are full row and full column rank, respectively, to ensure the existence of the general solution (43). 
Assumption 2 (see [34])

$$
\left\{\begin{array}{l}
\left|\dot{\rho}_{q}(\xi(t))\right| \leq \lambda_{q} \\
\lambda_{q} \geq 0
\end{array}\right.
$$

Lemma 1 (see [35]). Let $\mathbf{A}, \Upsilon, \mathbf{P}$, and $\Theta$ be matrices with proper sizes. The following two inequalities are equivalent:

$$
\left.\begin{array}{cc}
\Theta+A^{T}+P A<0, \\
A^{T} \Upsilon^{T}+\Upsilon A+\Theta & P-\Upsilon+A^{T} \Upsilon^{T} \\
* & -\Upsilon-\Upsilon^{T}
\end{array}\right]<0 .
$$

Lemma 2 (see [36]). Considering the matrix $\mathbb{W} \varepsilon \mathbb{R}^{\mathbf{n} \times \mathbf{m}}$, with $\mathbf{n} \geq \mathbf{m}$, and matrix $\mathbb{Y} \in \mathbb{R}^{\mathbf{n} \times \mathbf{k}}$, the matrix $\mathbb{X}$ with the form

$$
\mathbb{X}=\mathbb{Y W}^{+}+\mathbb{U}\left(I-\mathbb{W W}^{+}\right),
$$

is a solution of $\mathbb{X W}=\mathbb{Y}$ when the condition $\mathbb{Y} \mathbb{W}^{+} \mathbb{W}=\mathbb{Y}$ holds. $\mathbb{U} \in \mathbb{R}^{k \times m}$ is an arbitrary matrix, and $\mathbb{W}^{+}$is the Moore-Penrose pseudoinverse of $\mathrm{W}$ which is denoted as

$$
\mathbb{W}^{+}=\left(\mathbb{W}^{T} \mathbb{W}\right)^{-1} \mathbb{W}^{T}
$$

As mentioned in the introduction, the next section will focus on actuator/sensor faults and state estimation. The block diagram shown in Figure 2 introduces the concept of the unknown input observer, where $u(t)$ is the input, $f(t)$ is the fault signal, $\hat{x}(t)$ and $\widehat{f}(t)$ are the state and the fault est $i$ mation, respectively, and $y(t)$ is the system output.

\section{Stability Analysis and Design of a Relaxed Unknown Input Observer}

Presently, there are several practical thoughtfulness in the field of vehicle production that inhibit using sensors, in particular, lateral velocity and yaw rate sensors, such as high cost, degradation, or loss of signal during certain weather or other conditions. To overcome this problem, we can use the observer theory. In this section, we aim to design an unknown input observer to estimate simultaneously the vehicle state and the sensor/actuator faults, despite the presence of uncertainties affecting both the state and the input matrices.

Let us consider the following observer which has the structure same as that of the previous T-S fuzzy system:

$$
\left\{\begin{array}{l}
\dot{p}(t)=\sum_{i=1}^{2} \rho_{i}(\xi(t))\left\{N_{i} p(t)+G_{i} u(t)+L_{i} y(t)\right\}, \\
\widehat{z}(t)=p(t)-H y(t) .
\end{array}\right.
$$

$N_{i}, G_{i}, L_{i}$, and $H$ are the observer's parameters to be determined. $p(t)$ and $\widehat{z}(t)$ are the observer states and augmented system estimation, respectively. The error between the faulty system (15) and the observer (20) is given by

$$
\begin{aligned}
e(t) & =\widehat{z}(t)-z(t) \\
& =p(t)-(I+H \bar{C}) z(t) \\
& =p(t)-T z(t) .
\end{aligned}
$$

The dynamic of the estimation error (21) is written by the following equation:

$$
\begin{aligned}
\dot{e}(t) & =\dot{p}(t)-T \dot{z}(t) \\
& =\sum_{i=1}^{2} \rho_{i}(\xi(t))\left\{N_{i} p(t)+G_{i} u(t)+L_{i} y(t)-T \bar{A}_{i} z(t)-T \bar{B}_{i} u(t)-T \bar{M} \omega_{i}(t)\right\} \\
& =\sum_{i=1}^{2} \rho_{i}(\xi(t))\left\{\left(N_{i} T+L_{i} \bar{C}-T \bar{A}_{i}\right) z(t)+\left(G_{i}-T \bar{B}_{i}\right) u(t)-T \bar{M} \omega_{i}(t)+N_{i} e(t)\right\} .
\end{aligned}
$$

If the conditions (23a)-(23c) are satisfied,

$$
\begin{aligned}
N_{i} T+L_{i} \bar{C}-T \bar{A}_{i} & =0, \\
G_{i}-T \bar{B}_{i} & =0, \\
T \bar{M} & =0 .
\end{aligned}
$$

Then, (22) is rewritten as follows:

$$
\dot{e}(t)=\sum_{i=1}^{2} \rho_{i}(\xi(t)) N_{i} e(t) .
$$

Theorem 1 presents the condition that system (24) is asymptotically stable.
Theorem 1. For given positive scalars $\lambda_{\mathbf{k}}$ and matrices $\mathbf{N}_{\mathbf{i}}, \mathbf{G}_{\mathbf{i}}$, $\mathbf{L}_{\mathbf{i}}$, and $\mathbf{H}$, the error system (24) is asymptotically stable if there exist positive symmetric matrices $\mathbf{P}_{\mathbf{i}}(\mathbf{i}=1,2)$ such that (25)-(27) hold.

$$
\sum_{k=1}^{2} \lambda_{k} P_{k}+N_{i}^{T} P_{i}+P_{i} N_{i}<\Gamma_{i i}
$$

$$
\begin{gathered}
\sum_{k=1}^{2} \lambda_{k} P_{k}+N_{i}^{T} P_{j}+P_{j} N_{i}+N_{j}^{T} P_{i}+P_{i} N_{j} \leq \Gamma_{i j}+\Gamma_{i j}^{T}, \\
{\left[\begin{array}{cc}
\Gamma_{11} & \Gamma_{12} \\
* & \Gamma_{22}
\end{array}\right]<0, \quad i, j=1,2 i<j .}
\end{gathered}
$$




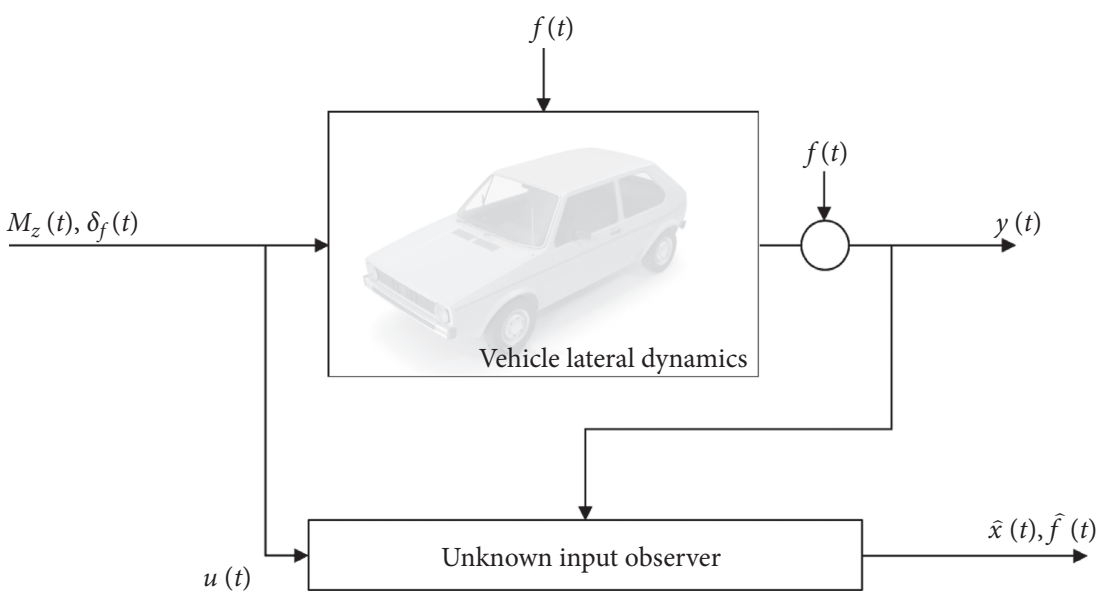

FIGURE 2: Observation scheme.

Proof. Let us consider the following Lyapunov function candidate:

$$
V(t)=\sum_{i=1}^{2} \rho_{i}(\xi(t)) e^{T}(t) P_{i} e(t),
$$

where $P_{i}=P_{i}^{T}>0$. Taking the time derivative of $V(t)$, we obtain

$$
\begin{aligned}
\dot{V}(t)= & \sum_{i=1}^{2} \dot{\rho}_{i}(\xi(t)) e^{T}(t) P_{i} e(t) \\
& +\sum_{i=1}^{2} \rho_{i}(\xi(t))\left\{\dot{e}^{T}(t) P_{i} e(t)+e^{T}(t) P_{i} \dot{e}(t)\right\} .
\end{aligned}
$$

Substituting (24) into (28), we get

$$
\dot{V}(t)=\sum_{i=1}^{2} \sum_{j=1}^{2} \rho_{i}(\xi(t)) \rho_{j}(\xi(t))\left\{\sum_{k=1}^{2} \dot{\rho}_{k}(\xi(t)) e^{T}(t) P_{k} e(t)+e^{T}(t)\left(N_{i}^{T} P_{j}+P_{j} N_{i}\right) e(t)\right\}
$$

By using the condition of Assumption 2, we obtain $\dot{V}(t) \leq \sum_{i=1}^{2} \sum_{j=1}^{2} \rho_{i}(\xi(t)) \rho_{j}(\xi(t)) e^{T}(t)\left\{\sum_{k=1}^{2} \lambda_{k} P_{k}+N_{i}^{T} P_{j}+P_{j} N_{i}\right\} e(t)$
By using Lemma 1 of [35] for (31), we have

$$
\dot{V}(t) \leq e^{T}(t) \sum_{i=1}^{2} \rho_{i}^{2}(\xi(t))\left\{\sum_{k=1}^{2} \lambda_{k} P_{k}+N_{i}^{T} P_{i}+P_{i} N_{i}\right\}+\sum_{i=1}^{2} \sum_{i<j}^{2} \rho_{i}(\xi(t)) \rho_{j}(\xi(t))\left\{2 \sum_{k=1}^{2} \lambda_{k} P_{k}+N_{i}^{T} P_{j}+P_{j} N_{i}+N_{j}^{T} P_{i}+P_{i} N_{j}\right\} e(t) .
$$

If conditions in (25)-(27) are satisfied, it means that $\dot{V}(t)<0$, and this completes the proof.

Remark 1. The present result provides an improved extension of the work presented in [22]. Our work presents less conservative results, thanks to relaxation matrices involved via Lemma 1 of [35]. Furthermore, if we put $\Gamma_{\mathrm{ii}}=0$, we get the conditions in [22]. Thus, the proposed Theorem 1 is a general case of the results given by [22].

Theorem 2 provides the conditions for the asymptotic stability of the estimation error in (22).

Theorem 2. For given positive scalars $\lambda_{\mathrm{k}}$, the states and faults of system (15) are estimated asymptotically with observer (20) 
if there exist the matrices $\mathbf{N}_{\mathbf{i}}, \mathbf{G}_{\mathbf{i}}, \mathbf{L}_{\mathbf{i}}, \mathbf{H}, \mathbf{R}, \bar{Y}$, and $\bar{Q}_{\mathbf{i}}$ and positive symmetric matrix $\mathbf{P}_{\mathbf{i}}$ such that (33)-(35) hold.

$$
\begin{gathered}
{\left[\begin{array}{cc}
\Xi_{i i} & P_{i}-R+\Lambda_{i} \\
* & -R-R^{T}
\end{array}\right]<0, \quad i=1,2,} \\
{\left[\begin{array}{cccc}
\Xi_{i j}+\Xi_{j i} & P_{i}-R+\Lambda_{i}+\Lambda_{j} & P_{i}-R+\Lambda_{j} & -R_{1}+\Lambda_{j} \\
* & -R-R^{T} & -R & -R_{2} \\
* & * & -R-R^{T} & -R_{1}-R^{T} \\
* & * & * & -R_{2}-R_{2}^{T}
\end{array}\right] \leq 0, \quad i, j=1,2 ; i<j,} \\
{\left[\begin{array}{cc}
\Gamma_{11} & \Gamma_{12} \\
* & \Gamma_{22}
\end{array}\right]<0,}
\end{gathered}
$$

with

$$
\Xi_{i j}=\sum_{k=1}^{2} \lambda_{k} P_{k}+R \widetilde{A}_{i}+\widetilde{A}_{j}^{T} R^{T}-\bar{C}^{T} \bar{Q}_{i}^{T}-\bar{Q}_{j} \bar{C}+\bar{Y} \widetilde{C}_{i}+\widetilde{C}_{j}^{T} \bar{Y}^{T}-\Gamma_{i j},
$$

$$
\Lambda_{i}=\widetilde{A}_{i}^{T} R^{T}-\bar{C}^{T} \bar{Q}_{i}^{T}+\widetilde{C}_{i}^{T} \bar{Y}^{T},
$$

$\widetilde{A}_{i}=(I+W \bar{C}) \bar{A}_{i}$,

$\widetilde{C}_{i}=U_{t} \bar{C} \bar{A}_{i}$

$$
\begin{aligned}
W & =-\bar{M}(\overline{C M})^{+}, \\
(\bar{C} \bar{M})^{+} & =\left((\overline{C M})^{T}(\overline{C M})\right)^{-1}(\overline{C M})^{T}, \\
U_{t} & =I-(\overline{C M})(\overline{C M})^{+} .
\end{aligned}
$$

The observer gains are obtained as follows:

$$
\begin{aligned}
Y & =R^{-1} \bar{Y}, \\
Q_{i} & =R^{-1} \bar{Q}_{i}, \\
H & =W+Y U_{t}, \\
T & =I+H \bar{C},
\end{aligned}
$$

$$
N_{i}=T \bar{A}_{i}-Q_{i} \bar{C}
$$

Proof. If conditions (25) in Theorem 1 hold, by applying Lemma 1, we directly obtain the conditions (33). The conditions (34) are obtained by using Lemma 1 twice. In the first step, we define variables indicated in Lemma 1 as

$$
\Theta=2 \sum_{k=1}^{2} \lambda_{k} P_{k}+N_{i}^{T} P_{j}+P_{j} N_{i}-\Gamma_{i j}-\Gamma_{i j}^{T} .
$$

If the conditions in (26) hold, that means it is equivalent to the following conditions:

$$
\left[\begin{array}{cc}
N_{j}^{T} R^{T}+R N_{j}+\Theta & P_{i}-R+N_{j}^{T} R^{T} \\
* & -R-R^{T}
\end{array}\right]<0 .
$$

In the second step, condition (46) can be written as (47). By applying Lemma 1, we directly obtain the conditions in (48).

$$
\begin{aligned}
& \underbrace{\left[\begin{array}{cc}
2 \sum_{k=1}^{2} \lambda_{k} P_{k}+N_{i}^{T} R^{T}+R N_{i}-\Gamma_{i j}-\Gamma_{i j}^{T} & P_{i}-R+N_{i}^{T} R^{T} \\
* & -R-R^{T}
\end{array}\right]}_{\theta}+\underbrace{\left[\begin{array}{cc}
N_{j}^{T} & 0 \\
0 & 0
\end{array}\right]}_{P} A^{T} \underbrace{\left[\begin{array}{cc}
P_{i} & 0 \\
0 & 0
\end{array}\right]}_{P}+\left[\begin{array}{cc}
P_{i} & 0 \\
0 & 0
\end{array}\right]\left[\begin{array}{cc}
N_{j} & 0 \\
0 & 0
\end{array}\right]<0, \\
& {\left[\begin{array}{cc}
\Theta+A^{T} \widetilde{R}^{T}+\widetilde{R} A & P-\widetilde{R}+A^{T} \widetilde{R}^{T} \\
* & -\widetilde{R}-\widetilde{R}^{T}
\end{array}\right]<0,}
\end{aligned}
$$


with $\widetilde{R}=\left[\begin{array}{ll}R & R_{1} \\ R & R_{2}\end{array}\right]$.

From (23a)-(23c), we have

$$
(I+H \bar{C}) \bar{M}=0 \Leftrightarrow H(\bar{C} \bar{M})=-\bar{M} .
$$

The general solution of (49) according to Assumption 1 and Lemma 2 is (40), where $Y$ is an arbitrary matrix with appropriate dimension. We put $Q_{i}=L_{i}+N_{i} H$, and we replace it in (23a)-(23c); we get

$$
N_{i}=T \bar{A}_{i}-Q_{i} \bar{C}
$$

Then, we can write

$$
L_{i}=Q_{i}(I+\bar{C} H)-T \bar{A}_{i} H .
$$

By combining (41) and (50), we find

$$
\begin{aligned}
N_{i} & =(I+H \bar{C}) \bar{A}_{i}-Q_{i} \bar{C}, \\
& =(I+W \bar{C}) \bar{A}_{i}+\left(Y U_{t} \bar{C}\right) \bar{A}_{i}-Q_{i} \bar{C}
\end{aligned}
$$

Replacing $N_{i}$ by its expression (52) and taking into account the $\bar{Y}=R Y$ and $\bar{Q}_{i}=R Q_{i}$ give the linear matrix inequalities (33) and (34). This completes the proof.

Remark 2. The given $\lambda_{\mathbf{k}}$ is determined based on Assumption 2 . The choice of $\lambda_{\mathbf{k}}$ is made arbitrarily in such a way that the conditions of Theorem 2 are feasible and taking into account the quality of simulation results.

Remark 3. In comparison with some works in the literature [37-39], which just focus on sensor faults or actuator faults, we have concentrated, in this paper, on the problem of estimating actuator and sensor faults together.

\section{Simulation Results}

To demonstrate the efficiency of the proposed observer to estimate the vehicle states and faults, we have carried out some simulations using the vehicle model (1) and MATLAB software. We take the longitudinal velocity as it is constant, $v_{x}=30 \mathrm{~ms}^{-1}$, we have considered the steering angle shown in Figure 3, and the values of other parameters are listed in Table 1 [40]. It should be mentioned that the simulated results are obtained without control, which means the yaw moment is equal to zero $\left(M_{z}(t)=0\right)$. Note that each $\left(A_{i}, C\right)$ is observable.

We have chosen to add a variant fault $f(t)$ to both the input and output of the vehicle dynamics system, which takes the following form:

$$
\left\{\begin{array}{l}
0.46 t-2, \quad 0 \leq t \leq 5 \\
1, \quad 5 \leq t \leq 10 \\
\sin (3 t), \quad t \geq 10
\end{array}\right.
$$

The parameter matrices of the faulty uncertain system of the vehicle lateral dynamics (13) are

$$
\begin{aligned}
A_{1} & =\left[\begin{array}{cc}
-1.3181 & -19.7744 \\
0.0997 & -3.0611
\end{array}\right], \\
A_{2} & =\left[\begin{array}{cc}
-0.1022 & -19.9994 \\
0.0054 & -0.2365
\end{array}\right], \\
B & =\left[\begin{array}{c}
0 \\
0.0004
\end{array}\right], \\
B_{f 1} & =\left[\begin{array}{l}
3.3122 \\
61.5683
\end{array}\right], \\
B_{f 2} & =\left[\begin{array}{cc}
0.2625 \\
4.8799
\end{array}\right], \\
C & =\left[\begin{array}{cc}
1 & 0 \\
0 & 1
\end{array}\right], \\
D_{1} & =D_{2}=\left[\begin{array}{ll}
1 & 1
\end{array}\right], \\
M & =\left[\begin{array}{cc}
0.1 & 0 \\
0 & 0.1
\end{array}\right], \\
F & =\left[\begin{array}{ll}
1 & 1
\end{array}\right] .
\end{aligned}
$$

Selecting $\lambda_{1}=0.764$ and $\lambda_{2}=0.542$ and solving LMIs of Theorem 2, by using Matlab LMI toolbox [29, 41], the following observer gains are recovered:

$$
\begin{aligned}
& N_{1}=\left[\begin{array}{ccc}
-1.5344 & 0.1534 & 0.0015 \\
0.4722 & -16.3259 & 0.4152 \\
-25.8258 & 776.9778 & -50.0720
\end{array}\right] \text {, } \\
& N_{2}=\left[\begin{array}{ccc}
-1.5296 & 0.0536 & 0.0005 \\
-0.0732 & -5.0153 & 0.4603 \\
6.6316 & 103.1824 & 50.0136
\end{array}\right] \text {, } \\
& G_{1}=\left[\begin{array}{cc}
0.000 & 0.000 \\
0.000 & 72.96 \\
-0.036 & -7296.07
\end{array}\right] \text {, } \\
& G_{2}=\left[\begin{array}{cc}
0.000 & 0.000 \\
0.000 & 11.371 \\
-0.036 & -1137.116
\end{array}\right] \text {, } \\
& L_{1}=\left[\begin{array}{cc}
0.000 & 0.000 \\
0.261 & 50.00 \\
-26.059 & 5000.00
\end{array}\right] \text {, } \\
& L_{2}=\left[\begin{array}{cc}
0.000 & 0.000 \\
-0.067 & 50.00 \\
6.678 & 5000.00
\end{array}\right] \text {, } \\
& H=\left[\begin{array}{cc}
-1 & 0 \\
0 & 0 \\
0 & -100
\end{array}\right] \text {. }
\end{aligned}
$$

The system of vehicle lateral dynamics was simulated selecting the following initial conditions: $x(t)=\left[\begin{array}{ll}0 & 0\end{array}\right]^{T}$ and $\widehat{z}(t)=\left[\begin{array}{lll}0.4 & 0.4 & -0.4\end{array}\right]^{T}$. 


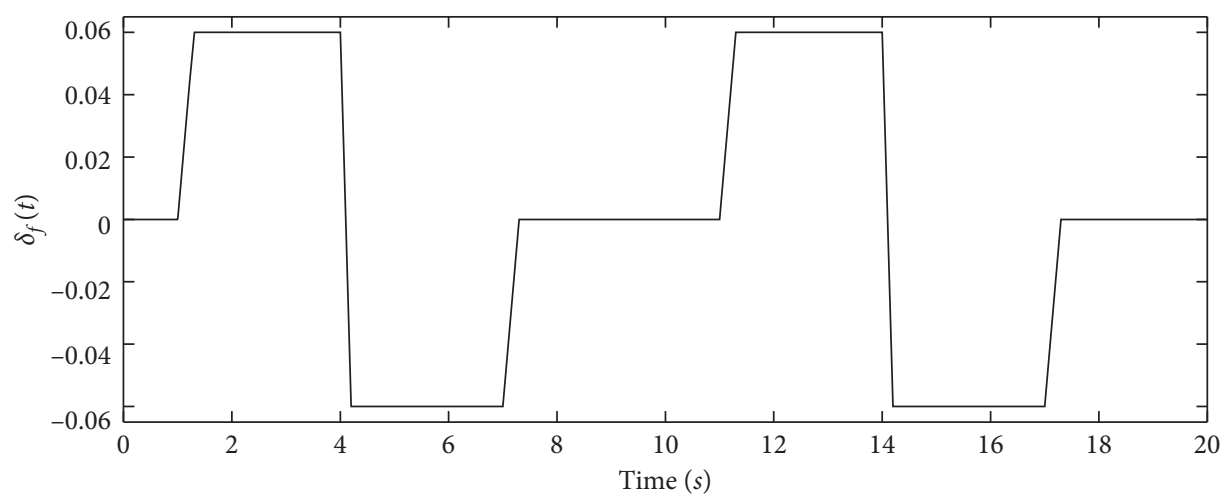

FIGURE 3: Profile of the steering angle given by driver $\delta_{f}(t)[\mathrm{rad}]$. Parameters of membership functions and stiffness coefficients are given in Table 2 [28].

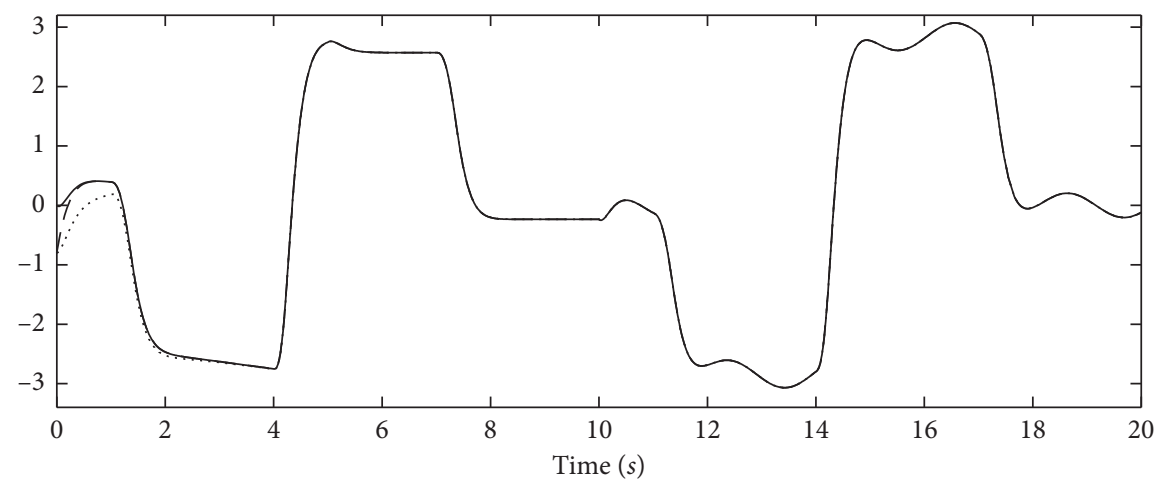

$$
\begin{aligned}
& -v_{y}(t) \\
& --\hat{v}_{y}(t) \text { by Theorem } 2 \\
& \ldots \ldots . . . \hat{v}_{y}(t) \text { by }[22]
\end{aligned}
$$

(a)

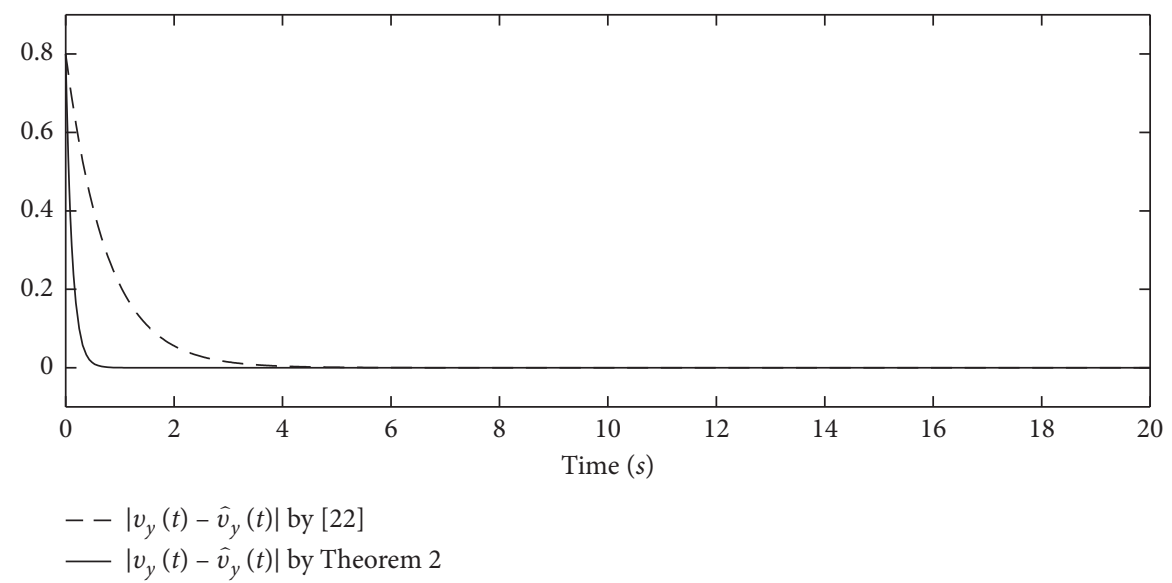

(b)

Figure 4: (a) Time evolution of lateral velocity and its estimates. (b) Error estimation of lateral velocity.

Figures 4 and 5 represent, respectively, the lateral velocity $v_{y}$ and the yaw rate $\dot{\psi}$ with their estimates in Figures 4(a) and 5(a) and estimation errors in Figures 4(b) and $5(\mathrm{~b})$, by simultaneously using the observers' gains recovered by Theorem 2 and the theorem of [22].
We can see, in both Figures 4 and 5, a relaxed estimation of states despite the presence of uncertainties and faults. Noticing that Theorem 2 presents the swiftness and the effectiveness compared to the theorem of [22] during estimation of vehicle lateral dynamics system states, this is 


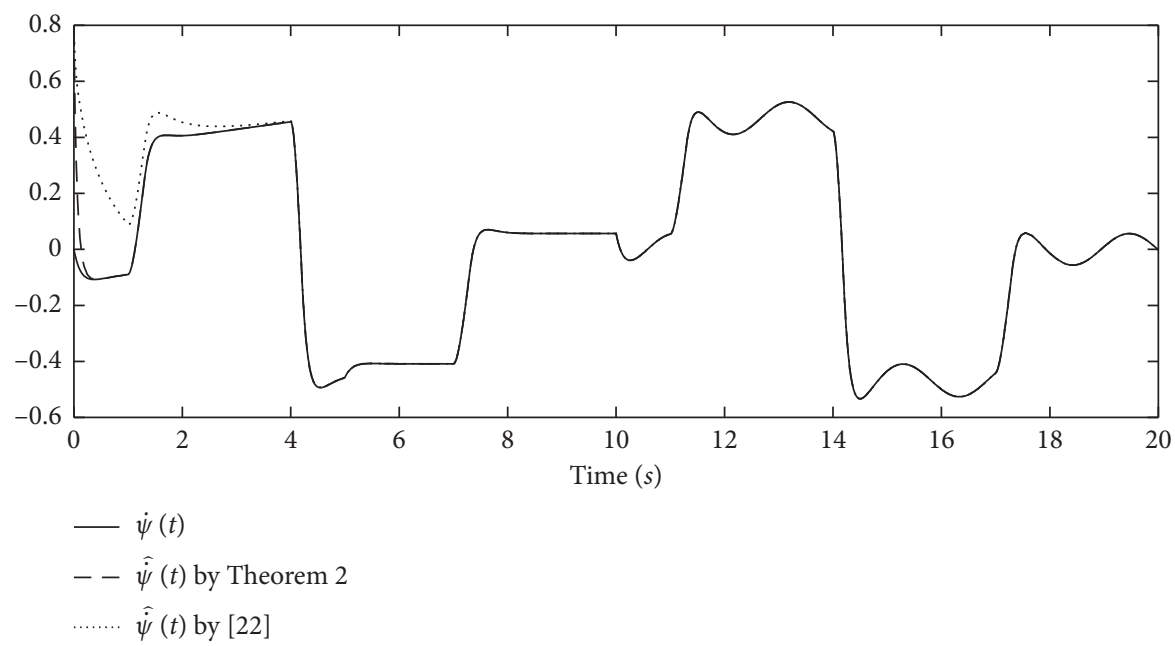

(a)

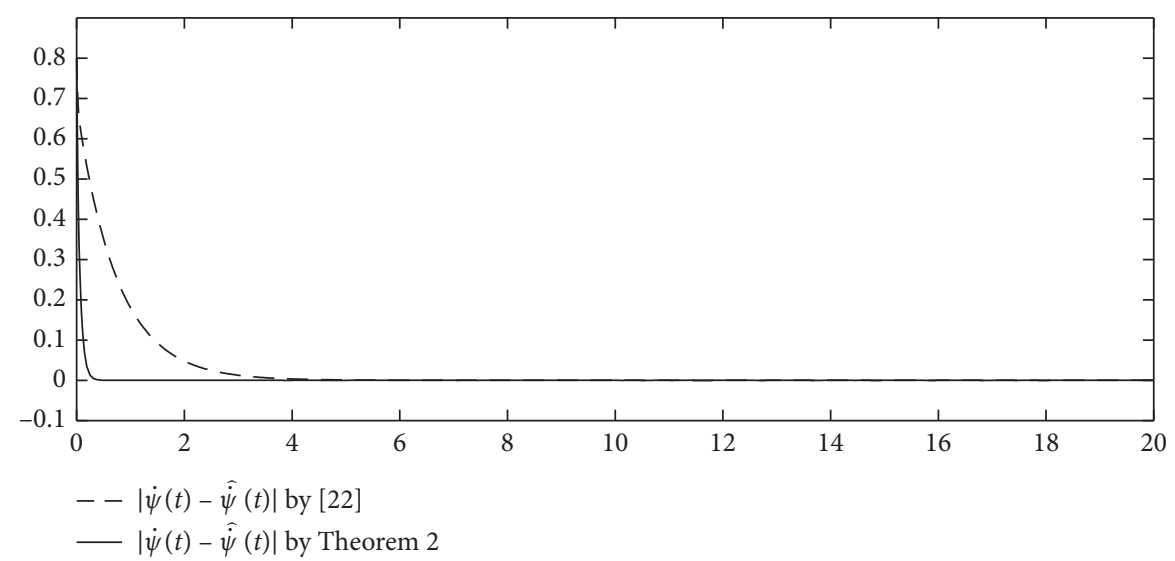

(b)

Figure 5: (a) Time evolution of the yaw rate and its estimates. (b) Error estimation of the yaw rate.

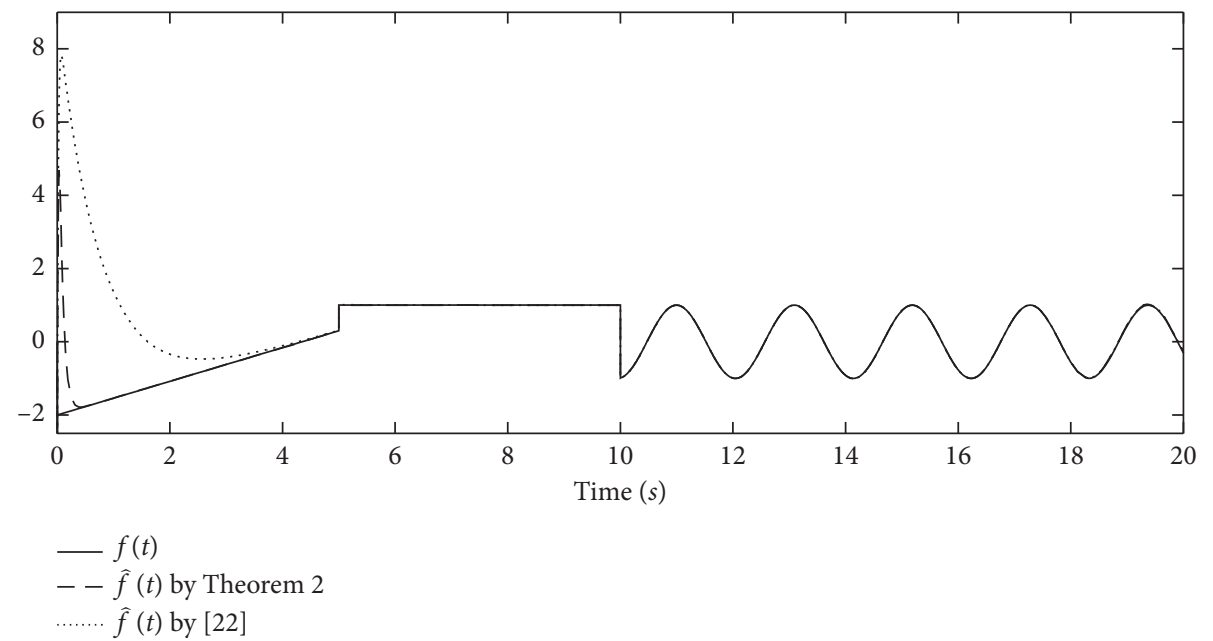

(a)

Figure 6: Continued. 


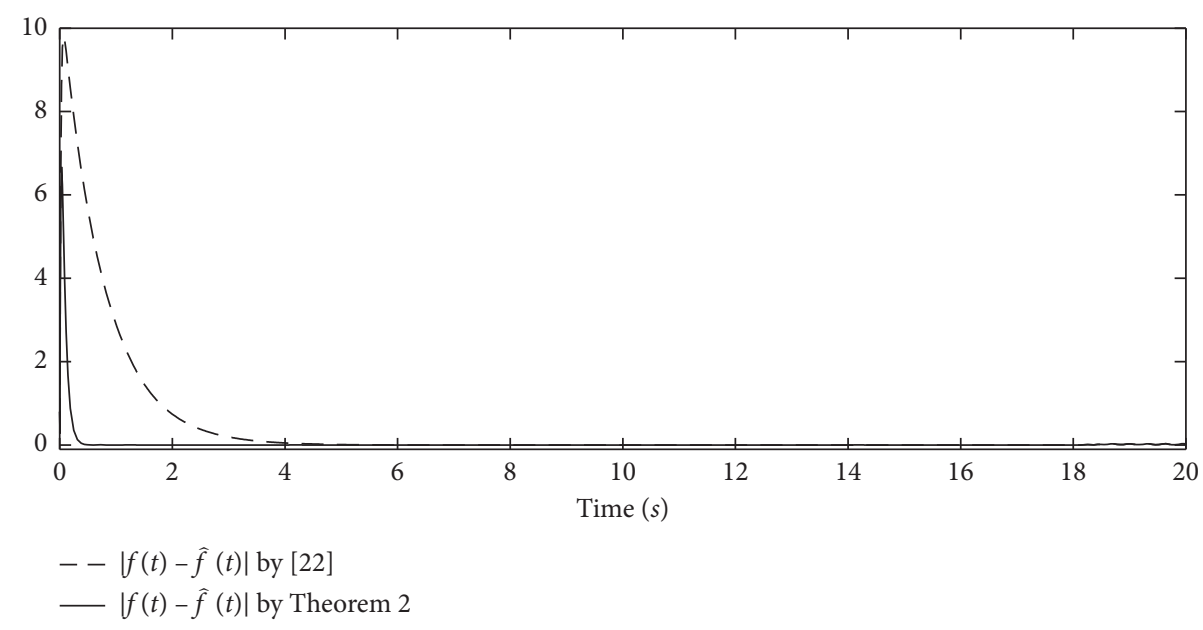

(b)

FIgURE 6: (a) Time evolution of fault and its estimates. (b) Error estimation of fault.

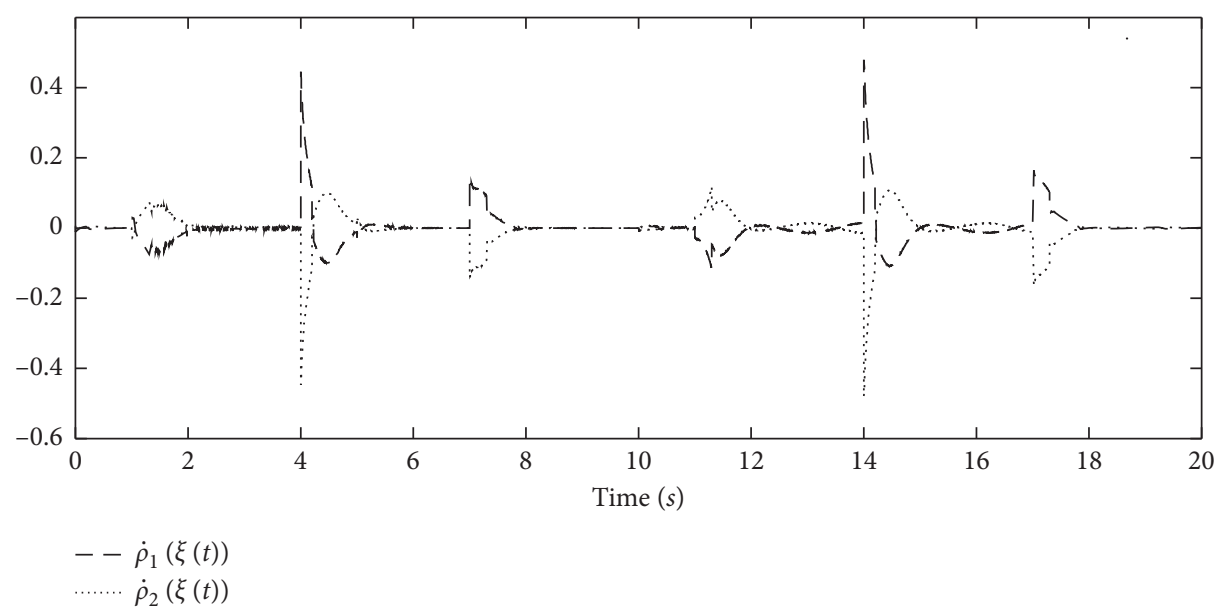

Figure 7: Membership functions derivative.

clearly seen in the evolution of the estimation errors (the solid line quickly converges to zero relative to the dotted one).

Figure 6 illustrates the fault added to the system and its estimates in Figure 6(a) and estimation errors in Figure 6(b), using different methods.

It is clear that the estimation by the observer's gains obtained by Theorem 2 converges rapidly towards the fault, just after it has appeared, faster than the result obtained by Theorem 2 of [22], and this is clearly seen in the evolution of the estimation errors (the solid line quickly converges to zero relative to the dotted one).

Figure 7 shows the membership function derivative $\dot{\rho}_{1}(\xi(t))$ and $\dot{\rho}_{2}(\xi(t))$ as considered in Assumption 2.

Remark 4. From Figure 7, we can see clearly that the conditions proposed in Assumption 2 are satisfied and the derivative of membership functions did not overcome its upper bound $\lambda_{1}$ and $\lambda_{1}$. This means that the proposed conditions respect its hypothesis.

\section{Discussion}

From these results, it can be deduced that the use of the proposed relaxed observer leads to much better results than those obtained by [22]. As mentioned in Remark 3, some works in the literature focus either on actuator or sensor faults and not both simultaneously. In this paper, both types of faults are investigated. However, the limitations of this study are reflected in the fact that an identical fault affects the actuator and the sensor at the same time, which is unusual in real systems, including the vehicle lateral dynamics system.

\section{Conclusions}

In this paper, we have proposed a relaxed unknown input observer to estimate actuator/sensors faults and states of an 
uncertain vehicle lateral dynamics system, which is represented by T-S fuzzy systems. The impacts of the uncertainties are absolutely removed, and the designed observers are asymptotically estimating the unmeasurable states and disturbances simultaneously. In order to compare the results obtained with the other results, the coincidence of the estimation error to zero is studied with the Lyapunov approach and LMI constraints, which are provided to design the matrices of the different components of the unknown input observer. The vehicle simulations show clearly the quality of faults and states estimation of the vehicle dynamics, and the proposed approach can be adapted to driving conditions. As the next perspective, we want to work on the design of an observer that can, besides estimating the states, estimate the sensor faults and actuator faults separately and in different forms.

\section{Data Availability}

No data were used to support this study.

\section{Conflicts of Interest}

The authors declare that they have no conflicts of interest.

\section{Acknowledgments}

The authors would like to thank Mr El Youssfi Mohamed for the preparation of the Word version as well as the English improvement of the paper.

\section{References}

[1] J. E. Gieck, D. Weitzenhof, M. Prichard, and F. Smith, "The production HELP bumper: evolution, testing, and evaluation," in Proceedings of the SAE Technical Paper Series, 1974.

[2] G. Belingardi, B. A. Beyene, E. Koricho, and B. Martorana, "Alternative lightweight materials and component manufacturing technologies for vehicle frontal bumper beam," Composite Structures, vol. 120, pp. 483-495, 2015.

[3] R. Andréasson and C.-G. Bäeckströem, The Seat Belt: Swedish Research and Development for Global Automotive Safety, Kulturvárdskommittén Vattenfall AB, Stockholm, Sweden, 2000.

[4] Y. Håland, "The evolution of the three point seat belt from yesterday to tomorrow," in Proceedings of the International Research Council on the Biomechanics of Injury Conference, Madrid, Spain, September 2006.

[5] C. C. Clark and W. A. Young, "Car crash theory and tests of airbag bumper systems," SAE Transactions, vol. 104, no. 6, pp. 1865-1879, 1995.

[6] G. Mastinu and M. Ploechl, Road and Off-Road Vehicle System Dynamics Handbook, CRC Press, Boca Raton, FL, USA, 2014.

[7] L. Austin and D. Morrey, "Recent advances in antilock braking systems and traction control systems," Proceedings of the Institution of Mechanical Engineers, Part D: Journal of Automobile Engineering, vol. 214, no. 6, pp. 625-638, 2000.

[8] H. Saito, T. Matsushita, B. Pipkorn, and O. Boström, "Evaluation of frontal impact restraint system in integrated safety scenario using human body model with PID controlled active muscles," in Proceedings of the International Research
Council on Biomechanics of Injury Conference, Malaga, Spain, September 2016.

[9] S. A. Ferguson, "The effectiveness of electronic stability control in reducing real-world crashes: a literature review," Traffic Injury Prevention, vol. 8, no. 4, pp. 329-338, 2007.

[10] S. Yim, Y. Park, and K. Yi, "Design of active suspension and electronic stability program for rollover prevention," International Journal of Automotive Technology, vol. 11, no. 2, pp. 147-153, 2010.

[11] A. Park and G. Jakubauskas, "Improvement of road safety using passive and active intelligent vehicle safety systems," Transport, vol. 22, no. 4, pp. 284-289, 2007.

[12] A. Vahidi and A. Eskandarian, "Research advances in intelligent collision avoidance and adaptive cruise control," IEEE Transactions on Intelligent Transportation Systems, vol. 4, no. 3, pp. 143-153, 2003.

[13] C. Afri, A. Andrieu, L. Bako, and P. Dufour, "State and parameter estimation: a nonlinear Luenberger observer approach," IEEE Transactions on Automatic Control, vol. 62, no. 2, pp. 973-980, 2016.

[14] Y. Wang, R. Rajamani, and D. M. Bevly, "Observer design for parameter varying differentiable nonlinear systems, with application to slip angle estimation," IEEE Transactions on Automatic Control, vol. 62, no. 4, pp. 1940-1945, 2016.

[15] D. Simon, "Training fuzzy systems with the extended Kalman filter," Fuzzy sets and systems, vol. 132, pp. 189-199, 2002.

[16] C. K. Chui and G. Chen, Kalman Filtering, Springer, Cham, Switzerland, 2017.

[17] R. Palm and P. Bergsten, "Sliding mode observer for a Takagi Sugeno fuzzy system," in Proceedings of the IEEE international conference on fuzzy systems, FUZZ-IEEE, vol. 2, pp. 665-670, San Antonio, TX, USA, May 2000.

[18] S. Yin, H. Gao, J. Qiu, and O. Kaynak, "Descriptor reducedorder sliding mode observers design for switched systems with sensor and actuator faults," Automatica, vol. 76, pp. 282-292, 2017.

[19] H. Geng, Y. Liang, F. Yang, L. Xu, and Q. Pan, “The joint optimal filtering and fault detection for multi-rate sensor fusion under unknown inputs," Information Fusion, vol. 29, pp. 57-67, 2016.

[20] N. E. Youssfi, M. Oudghiri, and R. E. Bachtiri, "Vehicle lateral dynamics estimation using unknown input observer," Procedia Computer Science, vol. 148, pp. 502-511, 2019.

[21] D. Rotondo, M. Witczak, V. Puig, and M. Pazera, "Robust unknown input observer for state and fault estimation in discrete-time Takagi-Sugeno systems," International Journal of Systems Science, vol. 47, no. 14, pp. 3409-3424, 2016.

[22] V.-P. Nejjari and T. D. Do, "Fault/state estimation observer synthesis for uncertain T-S fuzzy systems," IEEE Access, vol. 7, pp. 358-369, 2018.

[23] R. Rajamani, Vehicle Dynamics and Control, Springer Science \& Business Media, Boston, MA, USA, 2012.

[24] H. O. Wang, K. Tanaka, and M. F. Griffin, "An approach to fuzzy control of nonlinear systems: stability and design issues," IEEE Transactions on Fuzzy Systems, vol. 4, no. 1, pp. 14-23, 1996.

[25] X. Liu and Q. Zhang, "New approaches to $H_{\infty}$ controller designs based on fuzzy observers for T-S fuzzy systems Via LMI," Automatica, vol. 39, no. 3, pp. 1571-1582, 2003.

[26] J. Park, J. Kim, and D. Park, "LMI-based design of stabilizing fuzzy controllers for nonlinear systems described by TakagiSugeno fuzzy model," Fuzzy Sets and Systems, vol. 122, no. 1, pp. 73-82, 2001. 
[27] N. El Youssfi, R. El Bachtiri, and H. El Aiss, "T-S fuzzy observers design and actuator fault tolerant control applied to vehicle lateral dynamics," International Journal of Digital Signals and Smart Systems, In press, 2020.

[28] N. El Youssfi, R. El Bachtiri, R. Chaibi, and E. H. Tissir, "static output-feedback H_infty control for T-S fuzzy vehicle lateral dynamics," SN Applied Sciences, vol. 2, no. 1, p. 101, 2020.

[29] P. Gahinet, A. Nemirovskii, A. J. Laub, and M. Chilali, "The LMI control toolbox," in Proceedings of the 1994 33rd IEEE Conference on Decision and Control, vol. 3, pp. 2038-2041, Lake Buena Vista, FL, USA, December 1994.

[30] D. Schramm, M. Hiller, and R. Bardini, "Model of a typical complex complete vehicle," Vehicle Dynamics, Springer, Berlin, Germany, pp. 351-371, 2018.

[31] T. Taniguchi, K. Tanaka, H. Ohtake, and H. O. Wang, "Model construction, rule reduction, and robust compensation for generalized form of Takagi-Sugeno fuzzy systems," IEEE Transactions on Fuzzy Systems, vol. 9, no. 4, pp. 525-538, 2001.

[32] D. Ohtake, H. Arioui, and S. Mammar, "Observer design for two-wheeled vehicle: a Takagi-Sugeno approach with unmeasurable premise variables," in Proceedings of the 19th Mediterranean Conference on Control \& Automation (MED), pp. 934-939, Corfu, Greece, June 2011.

[33] N. El Youssfi and R. El Bachtiri, "T-S fuzzy observers to design actuator fault-tolerant control for automotive vehicle lateral dynamics," Building Transportation, Traffic and Engineering Systems, IntechOpen, London, UK, 2020.

[34] L. Zhao, H. Gao, and H. R. Karimi, "Robust stability and stabilization of uncertain T-S fuzzy systems with time-varying delay: an input-output approach," IEEE Transactions on fuzzy systems, vol. 21, no. 5, pp. 883-897, 2012.

[35] B. Bede, "Fuzzy sets," Chez Mathematics of Fuzzy Sets and Fuzzy Logic, pp. 1-12, Springer, Berlin, Germany, 2013.

[36] J. A. Bærentzen, J. Gravesen, F. Anton, and H. Aanæs, Guide to Computational Geometry Processing: Foundations, Algorithms, and Methods, Springer Science \& Business Media, London, UK, 2012.

[37] H. Zhang, J. Han, Y. Wang, and X. Liu, "Sensor fault estimation of switched fuzzy systems with unknown input," IEEE Transactions on Fuzzy Systems, vol. 26, no. 3, pp. 1114-1124, 2017.

[38] S. Gómez-Peñate, G. Valencia-Palomo, F.-R. López-Estrada, C.-M. Astorga-Zaragoza, R. A. Osornio-Rios, and I. SantosRuiz, "Sensor fault diagnosis based on a sliding mode and unknown input observer for takagi-sugeno systems with uncertain premise variables," Asian Journal of Control, vol. 21, no. 1, pp. 339-353, 2019.

[39] C. Martínez-García, V. Puig, C.-M. Astorga-Zaragoza, G. Madrigal-Espinosa, and J. Reyes-Reyes, "Estimation of actuator and system faults via an unknown input interval observer for takagi-Sugeno systems," Processes, vol. 8, no. 1, p. 61, 2020.

[40] M. Zhu, H. Chen, and G. Xiong, "A model predictive speed tracking control approach for autonomous ground vehicles," Mechanical Systems and Signal Processing, vol. 87, pp. 138152, 2017.

[41] S. Chen, L. El Ghaoui, E. Feron, and V. Balakrishnan, Linear Matrix Inequalities in System and Control Theory, Society for Industrial and Applied Mathematics (SIAM), Philadelphia, PA, USA, 1994. 